\title{
Koebner Phenomenon in Juvenile Dermatomyositis
}

W. JAMES TIDWELL, MD, Fellow; JEFFREY P. CALLEN, MD, Professor, Division of Dermatology, Department of Medicine, University of Louisville School of Medicine, Louisville, Kentucky, USA. Address correspondence to Dr. W. James Tidwell, 3810 Springhurst Blvd., Louisville, Kentucky 40241, USA. E-mail: wjamestidwell@gmail.com. An ethics board approval is not needed for this nonidentifiable patient, according to the University of Louisville Division of Dermatology guidelines. J Rheumatol 2018;45:437; doi:10.3899/jrheum.170885

Koebner phenomenon is an isomorphic response seen in a variety of dermatoses such as psoriasis. Its key feature is the appearance of a new dermatosis in previously unaffected areas following trauma.

An 8-year-old girl presented with several months history of a worsening rash on her hands and face, pruritus, and proximal muscle weakness. At age 4, a diagnosis of juvenile dermatomyositis was first established by the presence of heliotrope rash, Gottron papules, proximal muscle weakness, and elevated creatine kinase. A skin biopsy at that time revealed an interface dermatitis. Now at age 18 , the patient has been receiving no therapy for $>18$ months and remains in remission. With treatment that included hydroxychloroquine, prednisone, and methotrexate, the eruption and muscle disease cleared. Physical examination at age 8 revealed ragged cuticles with periungual telangiectases, as well as erythematous to violaceous scaly papules and plaques over the bony prominences. The latter finding is known as Gottron papules (Figure 1) and are characteristic of dermatomyositis ${ }^{1}$. Her parents noted exacerbation with sunlight. In addition, a linear scaly plaque was observed on her dorsal hand, which is a classic example of the Koebner phenomenon ${ }^{2}$.

The Koebner phenomenon is typically observed in psoriasis and lichen planus and has been reported rarely in patients with cutaneous lupus erythematosus. The triggering event of the new dermatosis could be a rash that, once resolved, leaves a second dermatosis in response ${ }^{3}$. An example is the appearance of psoriasis in a dermatomal distribution following a herpes zoster infection. This should not be confused with pathergy, which is the development of papules or blisters following an induced injury with intradermal injection. Pathergy is most often seen in neutrophilic dermatoses such as Behçet disease. In this case, neither the child nor parents remembered the exact details of the inducing trauma, but it was thought to occur subsequent to scratching from the pruritus of dermatomyositis. To our knowledge, patients with dermatomyositis have not been previously reported to demonstrate the Koebner phenomenon.

\section{REFERENCES}

1. Callen JP. Cutaneous manifestations of dermatomyositis and their management. Curr Rheumatol Rep 2010;12:192-7.

2. Miller RA. The Koebner phenomenon. Int J Dermatol 1982; 21:192-7.

3. Camargo CM, Brotas AM, Ramos-e-Silva M, Carneiro S. Isomorphic phenomenon of Koebner: facts and controversies. Clin Dermatol 2013;31:741-9.

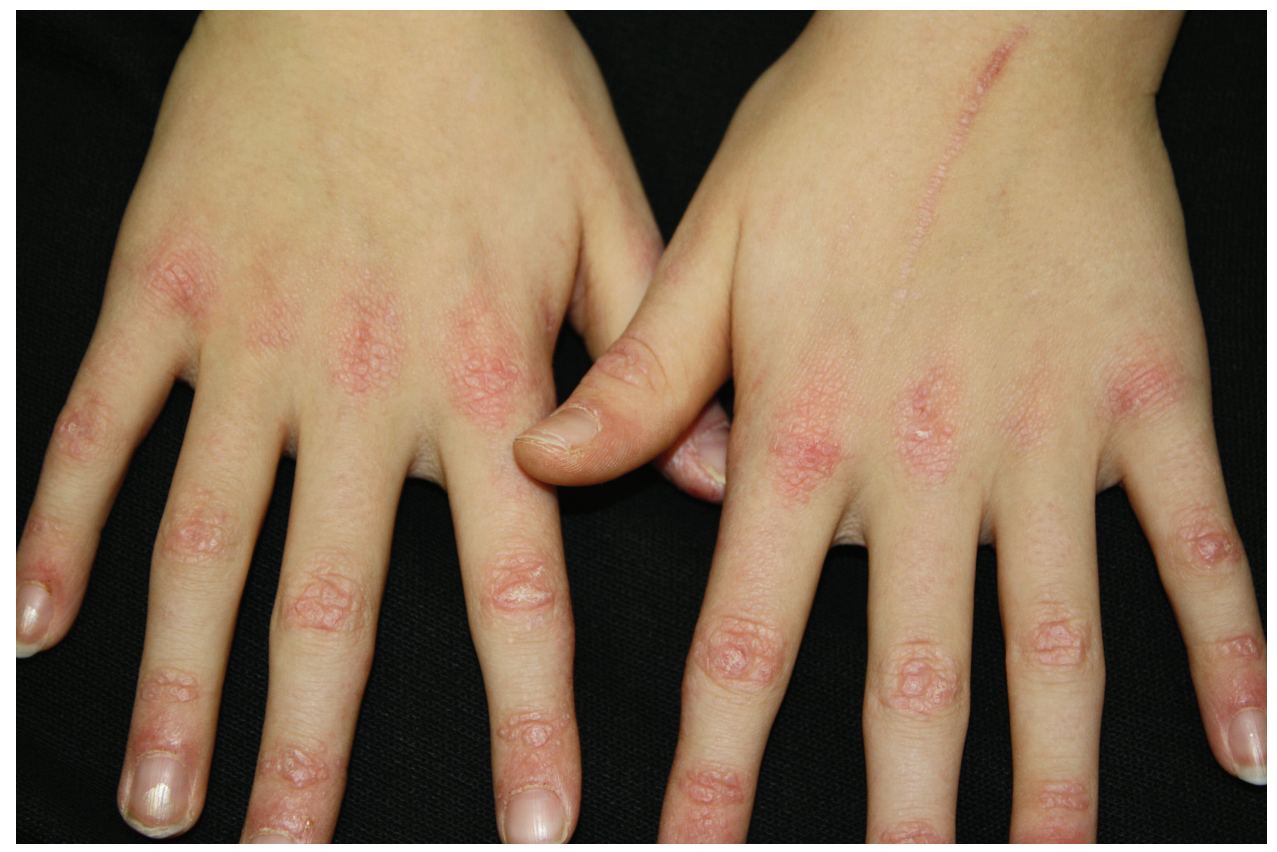

Figure 1. Classic Gottron papules seen in dermatomyositis with a rare isomorphic response noted on the dorsal hand. 